\title{
Beyond risk and ambiguity: Deciding under ignorance
}

\author{
Helen Pushkarskaya ANd Xun LiU \\ University of Kentucky, Lexington, Kentucky \\ Michael Smithson \\ Australian National University, Canberra, ACT, Australia \\ AND \\ JANE E. JosepH \\ University of Kentucky, Lexington, Kentucky
}

\begin{abstract}
In this study, we examined the neural basis of decision making under different types of uncertainty that involve missing information: ambiguity (vague probabilities) and sample space ignorance (SSI; unknown outcomes). fMRI revealed that these two different types of uncertainty recruit distinct neural substrates: Ambiguity recruits the left insula, whereas SSI recruits the anterior cingulate cortex, bilateral inferior parietal cortex, and the lateral orbitofrontal cortex. The finding of unique activations for different types of uncertainty may not necessarily be predicted within the reductive approach of modern theories of decision making under uncertainty, because these theories purport that humans reduce more complicated uncertain environments to subjectively formed less complicated ones (i.e., SSI to ambiguity). The predictions of the reductive view held only for ambiguityaverse individuals and not for ambiguity-tolerant individuals. Consequently, theories of decision making under uncertainty should include individual tolerance for missing information and how these individual differences modulate the neural systems engaged during decision making. Supplemental materials for this article may be downloaded from http://cabn.psychonomic-journals.org/content/supplemental.
\end{abstract}

Despite an emerging consensus that uncertainty modulates valuation (a process, or a combination of processes, of determining the current worth of the available options; Rangel, Camerer, \& Montague, 2008), most researchers focus only on two types of uncertainty: risk (known outcomes, known probabilities; von Neumann \& Morgenstern, 1944 ) and ambiguity (known outcomes, vague probabilities; Ellsberg, 1961). The subjective expected utility (SEU) theory, prospect theory (Tversky \& Kahneman, 1992), rank-dependent expected utility theory (Quiggin, 1993), and other theories treat unavailability of information as a negative characteristic of the environment and suggest that individuals reduce higher levels of uncertainty to risk by forming subjective beliefs about unknown characteristics of the environment (the reductive viewpoint) - for instance, reducing ambiguity to risk by forming subjective beliefs about the unknown probabilities. Behavioral studies have shown that individuals demonstrate ambiguity aversion (for a review, see Camerer \& Weber, 1992). Platt and Huettel (2008) suggested that parameters measuring the amount of ambiguity might be encoded in the brain (in the amygdala, the orbitofrontal cortex [OFC], or the anterior insula). Other types of uncertainty, including conflict (known outcomes, conflicting information about prob- abilities; Smithson, 1999), unawareness (decision makers are unaware of unknown outcomes; Modica \& Rustichini, 1994), and sample space ignorance (SSI; decision makers are aware of unknown outcomes; Smithson, Bartos, \& Takemura, 2000), still remain understudied. Unawareness is not likely to affect valuation (Modica \& Rustichini, 1999). However, conflict and SSI are as likely to modulate valuation as risk and ambiguity are, because under all these conditions decision makers are aware of the informational limitations during the valuation stage. To better understand how uncertainty modulates valuation, it is important to investigate the full variety of its types. It is also intriguing to examine whether individual preferences toward different types of uncertainty interact with their modulating effects. We begin this line of research by focusing the present article on uncertainties generated by the lack of information (ambiguity and SSI).

Baron and Frisch (1994) suggested that people regard any alternatives with missing information (MI) as inferior. Smithson et al. (2000) found that decision makers demonstrate aversion toward SSI. The reductive viewpoint suggests that individuals reduce SSI to ambiguity and then to risk by forming subjective beliefs, first about the partition of a sample space and then about the corresponding prob- 
Table 1

Brain Activation Patterns Predicted by the Reductive Approach and Three Alternative Hypotheses

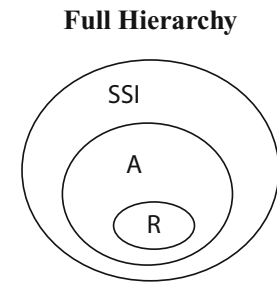

Logical Combinations
Partial Hierarchy

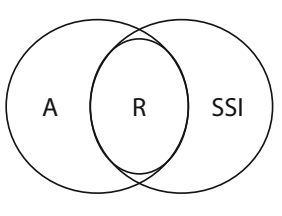

Partial Hierarchy With

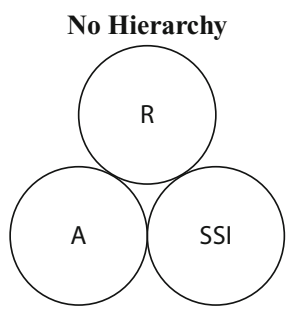

Missing Information

Predicted Activation

Unique activation for ambiguity (A):

A \& $\sim \mathrm{R} \& \sim \mathrm{SSI} \& \sim \mathrm{C}$

Unique activation for ignorance (SSI):

SSI \& $\sim \mathrm{R} \& \sim \mathrm{A} \& \sim \mathrm{C}$

Conjoined activation for A and SSI:

(A \& SSI) \& $\sim \mathrm{R} \& \sim \mathrm{C}$

$\begin{array}{cccc}\text { No } & \text { Yes } & \text { Yes } & \text { Yes } \\ \text { Yes } & \text { Yes } & \text { Yes } & \text { Yes } \\ \text { Yes/No } & \text { No } & \text { No } & \text { Yes }\end{array}$

Note-A, (partial) ambiguity; C, (partial) conflict; R, risk; SSI, (partial) sample space ignorance; MI, missing information. Activation associated with A was determined by isolating the voxels in which all of the following comparisons were statistically significant at $p<.05$ : A $>$ R, A $>$ SSI, $\mathrm{A}>\mathrm{C}, \mathrm{A}>$ baseline. Activation associated with SSI was determined by isolating the voxels in which all of the following comparisons were statistically significant at $p<.05$ : SSI $>$ R, SSI $>$ A, SSI $>$ C, SSI $>$ baseline. The conjoined activation for A and SSI was determined by the conjunction of the following contrasts: A $>$ R, A > C, A > baseline, SSI > R, SSI > C, SSI > baseline, with differential activation for A and SSI subtracted out. Note that the reductive viewpoint does not make a definite prediction about the A-SSI-conjoined activation. That is, neither the presence nor absence of A-SSI-conjoined activation would contradict it.

abilities. Overall, it implies that the two MI environments affect valuation in a similar manner, with SSI possibly evoking higher levels of aversion in decision makers than ambiguity.

The strong form of the reductive viewpoint therefore suggests a full hierarchy for brain systems associated with different types of uncertainty. That is, brain activation under risk should be subsumed by activation under ambiguity, which should be largely subsumed by activation under SSI (Table 1). The reductive viewpoint also implies that the main difference in brain activation patterns under ambiguity and SSI should be due to greater memory and calculation requirements under SSI (to create a subjective partition of a sample space before estimating probabilities and evaluating expected values, or utilities, of each choice). Higher calculation load during SSI may recruit the inferior parietal lobe (IPL), a region often implicated in numerical representation (Piazza \& Dehaene, 2004). Greater demands on working memory under SSI may recruit IPL and the dorsolateral prefrontal cortex (Wager \& Smith, 2003); limbic structures (the amygdala, the OFC, and the insula) may respond more strongly under SSI than under ambiguity because of the negative affect induced by higher levels of uncertainty under SSI.

In this article, we question whether the effect of MI on valuation is consistent with the reductive viewpoint and test the full-hierarchy hypothesis against three alternatives depicted in Table 1 in a simple gamble setting by isolating both conjoined and unique activations (Joseph, Partin, \& Jones, 2002) associated with each type of uncertainty. All three hypotheses predict some unique activation associated with SSI and ambiguity, contrary to the reductive viewpoint. These hypotheses differ on how much conjoined activation is expected among SSI, ambiguity, and risk. The no-hierarchy hypothesis predicts no conjoined activation for the three conditions. The partial-hierarchy hypothesis predicts that conjoined activation for SSI and ambiguity is also conjoined with activation for risk. Finally, the MI hypothesis predicts conjoined activation for SSI and ambiguity (attributed to MI processing) that is not fully conjoined with risk. We also examine whether brain activation patterns are stable with respect to individual preferences toward uncertainty by analyzing the relationship between conjoined and unique activations associated with each type of uncertainty and individual preferences toward ambiguity and SSI.

Note that we do not make any predictions about the conflict environment since the focus of the article is on the environments with MI, and not on environments with too much information. However, we included the conflict condition in our experiment and in the conjunction analyses because we wanted to isolate the brain regions that are more sensitive to unavailability of information (not to uncertainty in a general sense).

\section{METHOD}

\section{Participants}

Forty-two healthy right-handed participants (21 male, mean age $=24.6$ years) with normal or corrected-to-normal vision were compensated for participation. Data from 38 participants (20 male) with reasonable head motion (less than $1.7 \mathrm{~mm}$ ) were included in the analysis.

\section{Procedure and Stimuli}

Before the fMRI session, the participants saw a box filled with cards from various card games (over 1,000 cards from 18 different games, but the participants did not have this information) and were informed that they would play a series of lotteries (see Supplemental Material S-3). For each lottery, the experimenter selected 100 cards from the box, asked the participants to guess the type of card (e.g., Trivial Pursuit) that they would draw from this mixed deck. The par- 
ticipants could refuse to gamble and receive $\$ 3$ or play and receive $\$ 10$ if correct or nothing if incorrect.

The participants were informed that they would play lotteries under four different informational conditions. In each lottery, they had to choose between a sure payoff and a three-card gamble: one card of known type and known quantity and two cards forming risky, ambiguous, conflicting, or ignorance gambles. Risky (known types and quantities of all cards in the deck) and ambiguous (known types of cards in the deck but unknown composition of the cards) gambles were designed similarly to those in Hsu, Bhatt, Adolphs, Tranel, and Camerer (2005). However, for detailed discussion of the differences between the present study and the study by Hsu et al., see Supplemental Material S-1. In the conflict condition, the participants were given information about the quantities of types of cards in the deck from two conflicting sources. For instance, these sources agreed that there were exactly 20 Pokémon cards in the deck but disagree on how many tarot and Go Fish cards there were; Source A said that there were 30 tarot cards in the deck and 50 Go Fish cards, but Source B claimed that there were 50 tarot cards and 30 Go Fish cards. In the SSI condition, the participants were told that there were exactly 20 Pokémon cards and 80 other cards, but they were not given any information about the types of these other cards. Instead, the participants were given a suggestion to bet on a tarot card, even though there was no guarantee that the mixed deck contained any tarot cards. In this case, the participants could either choose a sure gain or choose to bet that they would draw from the deck a Pokémon card, a tarot card, or any other card.

The participants were told that they would play a series of similar gambles during fMRI scanning. They were also told that they would not be given any feedback while in the scanner on whether they had won or lost the gamble (because we were interested in the neurobiological processes associated with valuation). Instead, they were told that after the fMRI session, we would choose four gambles randomly (one from each condition), construct mixed decks of cards according to the description of the chosen gambles, and ask them to draw a card from each of the four decks. For each draw predicted correctly during the fMRI session, they would be paid $\$ 10$; for each incorrect prediction, they would not be paid; and for each choice of sure gain, they would receive $\$ 3$. In addition, they would be paid $\$ 10$ for their time. Overall, the participant payments ranged from $\$ 10$ to $\$ 50(M=\$ 38)$.

Before the $\mathrm{fMRI}$ session, the participants practiced on a computer (Figure 1 shows how each gamble was represented on the computer screen). During each trial, the participants had four options to choose from. The first option was a sure gain $(\$ 3)$. The second option was to bet on Card 1, which in all four gambles represented a risky option (known type and known quantity). The third option was to bet on Card 2, and the fourth option was to bet on Card 3.

In all risky gambles, the number of cards labeled Card 2 in the deck was equal to the number of those labeled Card 3; therefore, the probabilities of winning $\$ 10$ associated with Card 2 and Card 3 were equal in each risky gamble. Under ambiguity, Card 2 and Card 3 were informationally equivalent; therefore, following the ignorance prior hypothesis (Fox \& Rottenstreich, 2003), we hypothesized that the subjective probabilities of winning $\$ 10$ associated with Card 2 and Card 3 were equal in each ambiguity gamble. In the ignorance gamble, Card 2 represented a suggested card, and Card 3 represented any other card (an "everything else" outcome). We emphasized in our instructions (see Supplemental Material S-3) that the suggested card might not be in the deck or that only the suggested and the risky card (Card 1) would be in the deck. We hypothesized that because of our suggestion, the participants constructed a subjective partition consisting of three outcomes: a risky card, a suggested card, and a not risky or suggested card (consistent with the findings of Fox \& Rottenstreich, 2003). Since the probabilities both for the suggested card outcome and for the not suggested or risky card outcome ranged from 0 to 100 minus the number of risky cards, we hypothesized that subjective probabilities of winning $\$ 10$ associated with Card 2 and Card 3 were equal in each ignorance gamble.
All of the participants were quizzed on how well they understood the instructions (see Supplemental Material S-3). Only after they had answered all of the questions correctly were they invited to perform the task during fMRI scanning.

Note that the design described here models partial ambiguity and partial ignorance. We chose this design because full ignorance (all outcomes unknown) and full ambiguity (all probabilities unknown) are relatively rare in reality. Furthermore, the comparative ignorance hypothesis (Fox \& Tversky, 1995) suggests that this design should enforce ambiguity- and ignorance-averse choices (consistent with the reductive viewpoint). Finally, recall that we include a conflict condition in the experiment only to isolate brain regions that selectively respond to MI-for instance, to differentiate between processes associated with imprecise probabilities versus conflicting probabilities.

The participants underwent four fMRI scans ( 8 min each) in which all four types of gambles and a fixation cross were pseudorandomly ordered according to a simulation algorithm to maximize estimation efficiency (AFNI scripts available at http://afni.nimh .nih.gov/afni/doc/howto/3). Each gamble (or fixation cross) was on the screen for $6,500 \mathrm{msec}$, during which the participants chose among a sure gain, a risky card, and one of the two cards displaying a risky, ambiguous, ignorance, or conflict gamble. The interstimulus interval was 1,000 msec. All series were matched on SEU expected payoffs (Supplemental Tables S-1.1a-S-1.1d).

MRI data collection. A 3-tesla Siemens Trio MRI scanner with an eight-channel parallel head coil was used. For each run, 200 echoplanar images were acquired (2,500-msec TR, 30-msec TE, $81^{\circ}$ flip angle, 38 axial slices, $64 \times 64$ matrix, $3.5-\mathrm{mm}^{3}$ resolution). A T1weighted MPRAGE anatomical scan with $1-\mathrm{mm}^{3}$ voxels and a 1-min field-map scan were collected for each participant. Stimuli were presented using a high-resolution rear-projection system (Avotec, Stuart, FL) with buttonpresses recorded using a four-button fiber-optics response pad. E-Prime software (Psychology Software Tools, Pittsburgh, PA) controlled stimulus presentation and the recording of responses with each trial triggered by a scanner-generated optical pulse.

\section{Data Analysis}

Behavioral data. We recorded the participants' choices and the response time (RT). The effects of the trial order on the participants' choices and the RT were estimated using multivariate and univariate repeated measures ANOVAs and paired $t$ tests (see Supplemental Materials S-1c-S-1d). The participants' economic preferences for ambiguity $\left(\gamma_{1}\right)$ and SSI $\left(\gamma_{3}\right)$ were estimated via a choice model using Bayesian Markov chain Monte Carlo estimation (see Supplemental Material S-1e).

fMRI data. fMRI data preprocessing steps were completed using FSL's FEAT module (FMRIB's Software Library, www.fmrib .ox.ac.uk/fsl): motion correction (Jenkinson, Bannister, Brady, \& Smith, 2002); removal of a nonbrain tissue (Smith, 2002); spatial smoothing using a Gaussian 7-mm FWHM kernel; grand-mean intensity normalization of the 4-D data set by a single multiplicative factor; high-pass temporal filtering (Gaussian-weighted least-squares straight-line fitting, with $\sigma=25 \mathrm{sec}$ ). Customized onset timing (on or off) was generated according to the order of experimental conditions. The main regressors of interest (risk, ambiguity, SSI, conflict, bet, sure gain, and left- or right-hand buttonpress) were convolved with a double-gamma hemodynamic response function. Note that a regressor conflict was included in these analyses to isolate neurobiological responses associated selectively with $\mathrm{MI}$ environments.

The data were pooled over the four runs for individual participants. Spatially normalized contrast maps from individual participants were entered into mixed-effects group analysis. Each condition was contrasted with every other condition (e.g., ambiguity vs. risk, ambiguity vs. SSI, ambiguity vs. conflict, ambiguity vs. baseline) by combining group statistical maps using logical operations (Joseph et al., 2002; Table 1). The conjoined activation for ambiguity and SSI was determined using logical operators implemented in fslmaths by the conjunction of the following contrasts: ambigu- 
A

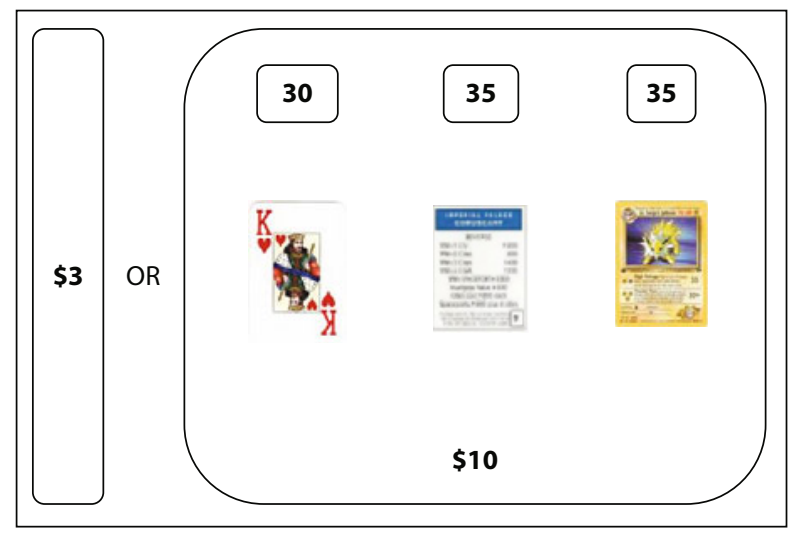

C

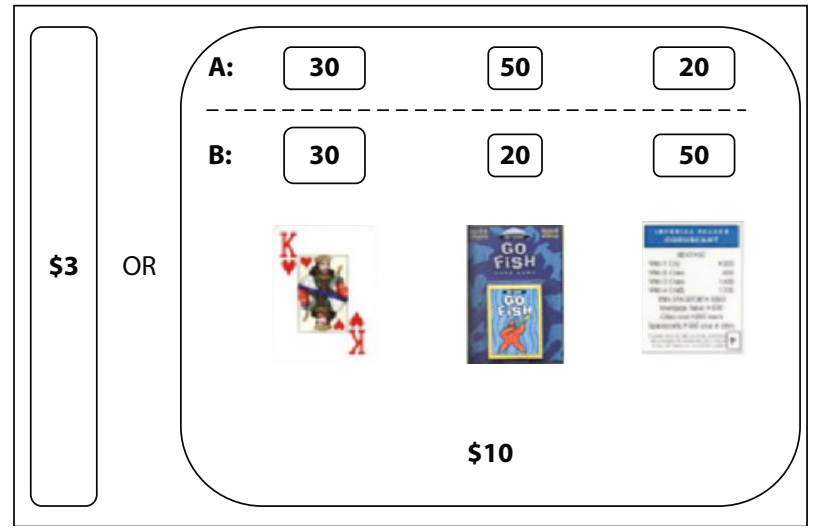

B

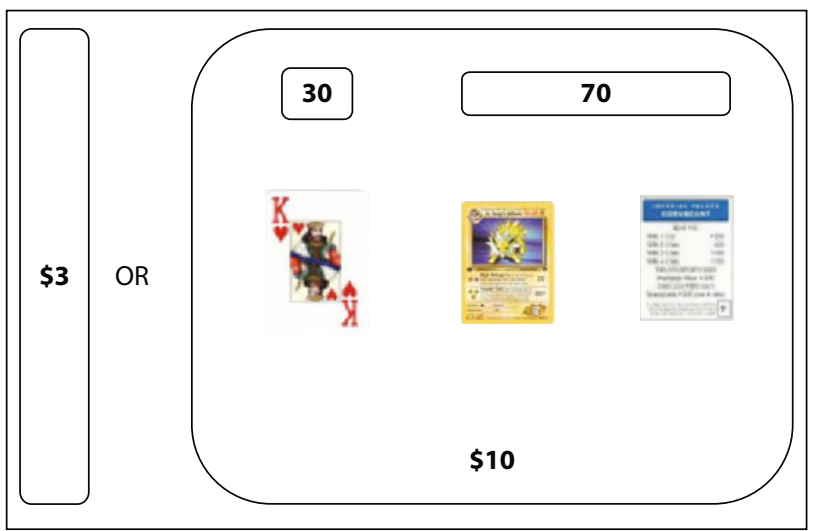

D

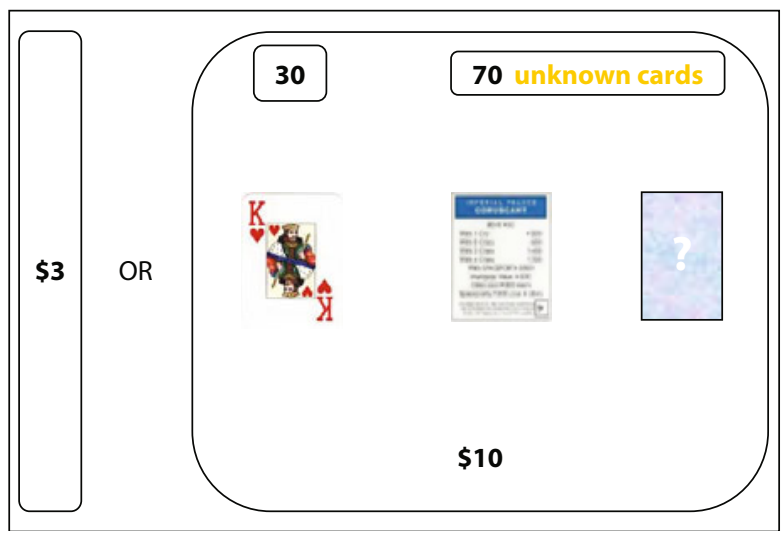

Figure 1. Sample stimuli used in all four experimental conditions. In each condition, participants choose between a certainty payoff and a three-card gamble - one card of known type and known quantity, and two cards forming risky, ambiguous, conflicting, or ignorance gambles. In the risk condition (A), participants were given information about types and quantities of cards in Piles 2 and 3 . In the ambiguity condition (B), participants were given information about types of cards in Piles 2 and 3 but only the total quantity of cards in Piles 2 and 3. In the conflict condition (C), participants were given information about types of cards in Piles 2 and 3 but conflicting information about the quantities of cards in each pile. In the sample space ignorance condition (D), participants were given information about the overall quantity of cards in Piles 2 and 3 but no information about possible card types in each pile. The participants were asked to treat a card type shown in the second pile as a suggestion and were told that there was no guarantee that this card would be included in the second or third pile.

ity $>$ risk, ambiguity $>$ conflict, ambiguity $>$ baseline, SSI $>$ risk, SSI $>$ conflict, SSI $>$ baseline, with differential activation for ambiguity and SSI subtracted out (Table 1). Recall that the conflict condition was included in these analyses to isolate neurobiological responses associated selectively with $\mathrm{MI}$ environments.

Region-of-interest analysis. We defined regions of interest (ROIs) as clusters of 27 or more contiguous voxels (Xiong, Gao, Lancaster, \& Fox, 1995) in which parameter estimate (PE) values differed significantly from zero ( $p<.05$, two-tailed). Although this cluster threshold appears lenient, recall that we used a combination of individual contrasts to construct the $z$ statistic maps for ambiguity-specific, SSI-specific, or ambiguity-SSI-conjoined activation, and only voxels that were significant at $p<.05$ for each of the individual contrasts were in the resulting $z$ statistic maps. Therefore, this approach is fairly conservative (Nichols, Brett, Andersson, Wager, \& Poline, 2005). Using the Mintun peak algorithm (Mintun, Fox, \& Raichle, 1989), we located the local peaks (maximal activation) within each ROI.

ROIs were identified without accounting for individual differences in attitudes toward ambiguity or SSI. Therefore, to understand whether individual economic preferences modulate the effect of various types of uncertainty on valuation, we evaluated whether $\log \gamma_{1}$ and $\log \gamma_{3}^{1}$ predicted activation under ambiguity $\left(\mathrm{PE}_{\mathrm{A}}\right)$, activation under SSI ( $\mathrm{PE}_{\mathrm{SSI}}$ ), and/or the difference between $\mathrm{PE}_{\mathrm{A}}$ and $\mathrm{PE}_{\mathrm{SSI}}$ $\left(\mathrm{PE}_{\mathrm{A}-\mathrm{SSI}}\right)$ in all ROIs. To that end, we employed a series of repeated measures ANCOVAs with the dependent variable $\mathrm{PE}_{\mathrm{A}}$ in ambiguityspecific and ambiguity-SSI-conjoined ROIs; the dependent variable $\mathrm{PE}_{\mathrm{SSI}}$ in SSI-specific and ambiguity-SSI-conjoined ROIs; and the dependent variable $\mathrm{PE}_{\mathrm{A}-\mathrm{SSI}}$ in ambiguity-specific, SSIspecific, and ambiguity-SSI-conjoined ROIs. For each ANCOVA, the within-subjects factor was condition-specific clusters (the levels of which depended on whether ambiguity-specific, SSI-specific, or ambiguity-SSI-conjoined clusters were analyzed), and the betweensubjects covariates were $\log \gamma_{1}$ (or $\log \gamma_{3}$ ) and selected personality measures (see Supplemental Material S-1b). In all analyses, we utilized the Greenhouse-Geisser statistic to choose between results of multivariate and univariate repeated measures ANCOVAs (Hertzog \& Rovine, 1985). Repeated measures ANCOVA provides more power than would separate analyses of each cluster to determine whether some clusters are associated with, for instance, $\log \gamma_{1}$ when 
AMBIGUITY (A) SPECIFIC:

$A \& \sim R \& \sim S S I \& \sim C$

\section{IGNORANCE (SSI) SPECIFIC:}

$S S I \& \sim R \& \sim A \& \sim C$

\section{AMBIGUITY AND IGNORANCE CONJOINED:}

( $A \& S S I) \& \sim R \& \sim C$
A

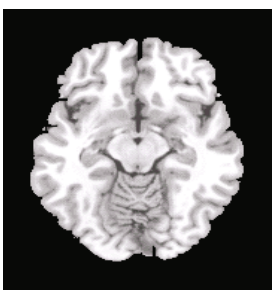

$\mathbf{B}$

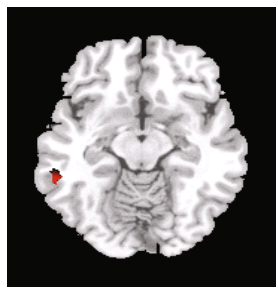

C

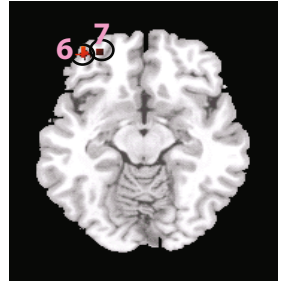

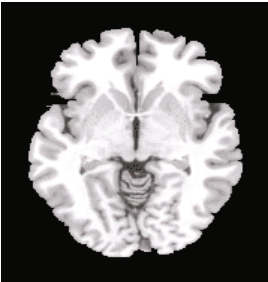
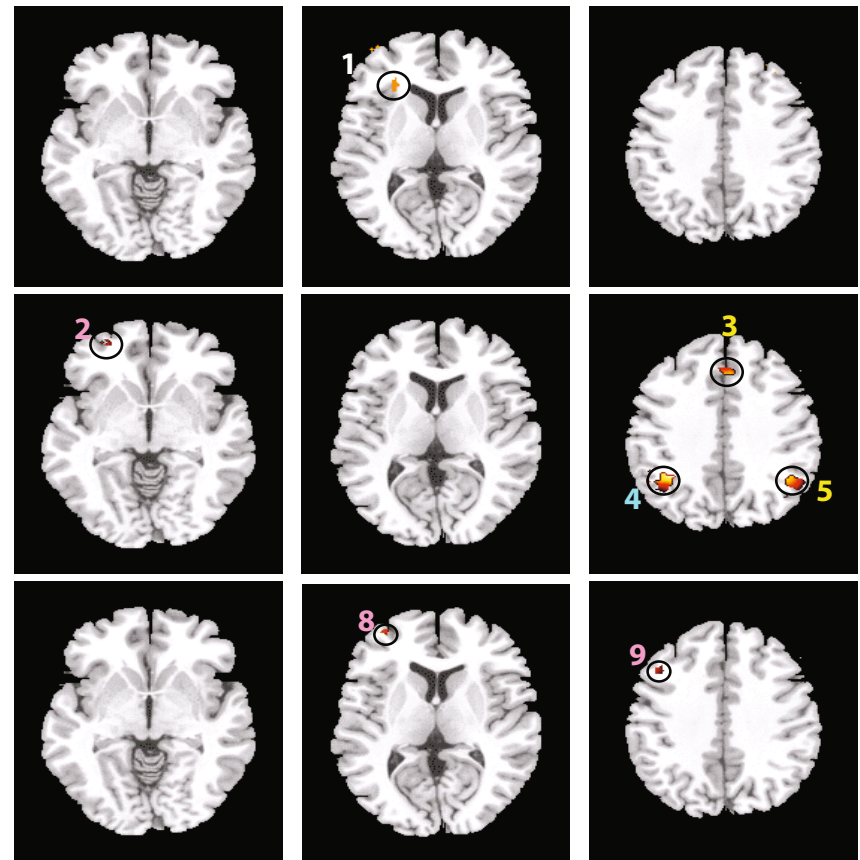

Figure 2. Statistical maps for (A) ambiguity-specific activation, (B) ignorance-specific activation, and (C) ambiguity-sample-spaceignorance (SSI) conjoined activation. The circled and numbered regions are highlighted in Table 2: (1) left insula (-26, 26, 4), (2) left lateral orbitofrontal $(-30,54,-4),(3)$ right anterior cingulate $(6,30,36),(4)$ left inferior parietal $(-36,-50,40),(5)$ right inferior parietal $(46,-50,44),(6)$ left inferior frontal $(-38,50,-12)$, (7) left middle orbitofrontal $(-28,52,-14)$, (8) left middle frontal $(-30$, $52,6),(9)$ left middle frontal $(-40,20,40)$.

others are not (through an interaction of cluster and behavioral or personality covariate), and it can identify significant variations in the correlations across clusters.

These additional analyses tested the hypothesis that individual preferences toward ambiguity (or SSI) do not modulate the effect of ambiguity and $\mathrm{SSI}$ on valuation (i.e., that $\mathrm{PE}_{\mathrm{A}}, \mathrm{PE}_{\mathrm{SSI}}$, and $\mathrm{PE}_{\mathrm{A}-\mathrm{SSI}}$ do not correlate significantly with individual economic preferences). In all clusters showing a main effect of ambiguity or ignorance aversion or an interaction effect involving ambiguity or ignorance aversion, a $95 \%$ confidence interval was generated for the regression lines $\mathrm{PE}_{\mathrm{A}-\mathrm{SSI}}$ versus $\log \gamma_{1}$ (or $\log \gamma_{3}$ ). If $95 \%$ confidence intervals for $\mathrm{PE}_{\mathrm{A}-\mathrm{SSI}}$ in ambiguity-specific and SSI-specific clusters do not include 0 for all individuals despite their economic preferences, it indicates a robust ambiguity-specific or SSI-specific network that is not modulated by preferences toward ambiguity (SSI). If the $95 \%$ confidence interval for $\mathrm{PE}_{\mathrm{A}-\mathrm{SSI}}$ in ambiguity-SSI-conjoined clusters includes 0 for all individuals despite their economic preferences, it indicates that a shared network for processing MI is not modulated by preferences toward ambiguity (SSI).

\section{RESULTS}

\section{Behavioral Measures}

Choice between Card 2 and Card 3. The results of our analyses (see Supplemental Material S-1c) were consistent with the hypothesis that the subjective probabilities of winning $\$ 10$ associated with choosing Card 2 and Card 3 are equal under ambiguity and SSI. Specifically, under ambiguity (SSI), Card 2 was chosen $13.5 \%$ (14.8\% under SSI) of the time and Card 3 was chosen $15.7 \%(17.5 \%)$ of the time. The relative frequencies of choices of each of the cards were not significantly differ- ent either under the ambiguity $[t(38)=-0.88, p=.385]$ or under the SSI $[t(38)=-0.718, p=.477]$ conditions; the differences in frequencies did not vary across conditions $[t(38)=-0.080, p=.937]$ and were stable across trials $[F(1,37)=0.24, p=.37]$.

Economic preferences. The means of $\gamma_{1}(0.964)$ and $\gamma_{3}(0.882)$ were close to $1\left[\gamma_{1}\left(\gamma_{3}\right)=1\right.$ implies a "rational" SEU agent, $\gamma_{1}\left(\gamma_{3}\right)<1$ implies tolerance toward ambiguity (SSI), and $\gamma_{1}\left(\gamma_{3}\right)>1$ implies aversion toward ambiguity (SSI)], did not differ significantly (the credible interval for the mean $\gamma_{3}-\gamma_{1}$ includes 0 ), and were strongly positively correlated $(r=.85)$.

RT. On average, RTs across conditions did not differ significantly. The mean RT under risk was $2.19 \mathrm{sec}(\Delta=$ $0.7 \mathrm{sec})$, that under ambiguity was $2.29 \mathrm{sec}(\Delta=0.6 \mathrm{sec})$, and that under SSI was $2.26 \sec (\Delta=0.5 \mathrm{sec})$.

\section{Brain Activation}

Risk did not produce greater activation than the other conditions, most likely because the risky gamble was present in each condition. Nevertheless, this outcome is consistent with all of the models except the no-hierarchy model. The presence of ambiguity-specific, SSI-specific, and ambiguity-SSI-conjoined regions (Figure 2, Table 2) indicates that uncertainties generated through MI modulate valuation differently than uncertainties generated through conflicting information. Six regions (located primarily in the frontal lobe) showed conjoined activation for ambiguity and SSI but were not associated with the risk condition, which rules out both the partial-hierarchy and no-hierarchy 
Table 2

Brain Activation Associated With Ambiguity and SSI

\begin{tabular}{|c|c|c|c|c|c|c|c|c|c|}
\hline \multirow[b]{2}{*}{ Region } & \multirow[b]{2}{*}{$x$} & \multirow[b]{2}{*}{$y$} & \multirow[b]{2}{*}{$z$} & \multirow[b]{2}{*}{ Size } & \multirow[b]{2}{*}{ BA } & \multirow[b]{2}{*}{$\operatorname{Max}(z)$} & \multicolumn{3}{|c|}{$\begin{array}{c}\text { Correlations With } \\
\text { Behavioral Parameters } \\
\text { and Selected Personality } \\
\text { Scales }\end{array}$} \\
\hline & & & & & & & $\mathrm{PE}_{\mathrm{A}}$ & $\mathrm{PE}_{\mathrm{SSI}}$ & $\mathrm{PE}_{\mathrm{A}-\mathrm{SSI}}$ \\
\hline \multicolumn{10}{|c|}{$\begin{array}{l}\text { Ambiguity-Specific Region } \\
\text { A \& } \sim \mathrm{R} \& \sim \text { SSI \& } \sim \mathrm{C}\end{array}$} \\
\hline Left insula & -26 & 26 & 4 & 42 & - & 4.42 & $-\mathrm{N}$ & $\mathrm{N} / \mathrm{A}$ & $-\gamma_{1}^{* a}$ \\
\hline \multicolumn{10}{|c|}{$\begin{array}{l}\text { SSI-Specific Regions } \\
\text { SSI \& } \sim \mathrm{R} \& \sim \mathrm{A} \& \sim \mathrm{C}\end{array}$} \\
\hline Right anterior cingulate & 6 & 30 & 36 & 35 & 32 & 6.98 & N/A & - & $-\gamma_{1}^{*}$ \\
\hline Right inferior parietal & 46 & -50 & 44 & 455 & 40 & 7.84 & $\mathrm{~N} / \mathrm{A}$ & - & $-\gamma_{1}^{*}$ \\
\hline Left lateral orbitofrontal & -30 & 54 & -4 & 37 & 11 & 5.40 & N/A & - & $-\gamma_{1}^{*}$ \\
\hline Left inferior parietal & -36 & -50 & 40 & 699 & 40 & 8.51 & N/A & - & $-\gamma_{1}^{*}$ \\
\hline \multicolumn{10}{|c|}{$\begin{array}{l}\text { Ambiguity-SSI-Conjoined Regions } \\
\text { (A \& SSI) \& } \sim \mathrm{R} \& \sim \mathrm{C}\end{array}$} \\
\hline Left fusiform & -36 & -52 & -18 & 48 & 37 & 7.63 & - & $+\gamma_{3}^{*}$ & $-\gamma_{1}^{* a}$ \\
\hline Left orbitofrontal & -28 & 52 & -14 & 29 & 11 & 4.67 & - & $+\gamma_{3}^{*}$ & $-\gamma_{1}^{* a}$ \\
\hline Left inferior frontal & -38 & 50 & -12 & 46 & 47 & 7.91 & - & $+\gamma_{3}^{*}$ & $-\gamma_{1}^{* a}$ \\
\hline Left middle frontal & -30 & 52 & 6 & 30 & 10 & 5.95 & - & $+\gamma_{3}^{*}$ & $-\gamma_{1}^{*}$ \\
\hline Right middle frontal & 44 & 22 & 42 & 31 & 44 & 5.30 & - & $+\gamma_{3}{ }^{*}$ & $-\gamma_{1}^{* a}$ \\
\hline Left middle frontal & -40 & 20 & 40 & 124 & 44 & 5.23 & - & $+\gamma_{3}^{*}$ & $-\gamma_{1}^{* a}$ \\
\hline
\end{tabular}

Note-BA, Brodmann's area; $\mathrm{PE}_{\mathrm{A}}$, activation under ambiguity; $\mathrm{PE}_{\mathrm{SSI}}$, activation under sample space ignorance; $\mathrm{PE}_{\mathrm{A}-\mathrm{SSI}}$, the difference between $\mathrm{PE}_{\mathrm{A}}$ and $\mathrm{PE}_{\mathrm{SSI}} ; \mathrm{N}$, neuroticism. ${ }^{*} \mathrm{Log}$ transformation. aCorrelation is marginal.

hypotheses but supports the MI hypothesis, positing unique brain systems associated with processing MI.

Ambiguity more strongly activated the left anterior insula (LaINS) than did the other conditions, which rules out the full-hierarchy model. Activation in the LaINS under the SSI and risk conditions did not differ statistically from the baseline activation $(p>.1)$.

SSI activated several regions more strongly than did the other conditions: a region in the left lateral OFC, broad areas in bilateral inferior parietal lobe (IPL), and a region in the left anterior cingulate cortex (ACC). Ambiguity activated the left IPL more strongly than did risk $[F(1,30)=$ $8.62, p=.006]$, which replicates Huettel, Stowe, Gordon, Warner, and Platt (2006).

\section{Effect of Individual Differences on Brain Activation Patterns}

The repeated measures ANCOVAs for ambiguity-SSIconjoined regions revealed that activation in these clusters under ambiguity did not correlate with $\log \gamma_{1}$, but positively correlated with $\log \gamma_{3}[F(1,36)=4.96, p=$ .032] under SSI. Because no interactions with clusters were significant in either analysis, all six ambiguitySSI-conjoined regions showed significant positive correlations with $\log \gamma_{3}$. The ANCOVA for the ambiguityspecific region revealed that activation under ambiguity in the LaINS was negatively associated with neuroticism $[F(1,32)=6.846, p=.013, r=-.42]$.

In all ROIs (ambiguity-specific, SSI-specific, and ambiguity-SSI-conjoined clusters) $\mathrm{PE}_{\mathrm{A}-\mathrm{SSI}}$ was negatively correlated with $\log \gamma_{1}$. In LaINS, where $\mathrm{PE}_{\mathrm{A}-\mathrm{SSI}}$ was positive, this correlation was marginally significant $[F(1,36)=3.37, p=.08]$. In the four SSI-specific clusters, where $\mathrm{PE}_{\mathrm{A}-\mathrm{SSI}}$ was negative, a main effect for $\log \gamma_{1}$ was significant $[F(1,36)=5.81, p=.02]$, but the interaction was not significant. In the six ambiguitySSI-conjoined clusters, the main effect was marginal $[F(1,36)=3.29, p=.08]$, but the interaction was not significant.

Since our analyses did not reveal any significant variations in the correlations across clusters in SSI-specific and ambiguity-SSI-conjoined regions, we generated $95 \%$ prediction bands only for the main effect of $\log \gamma_{1}$ on $\mathrm{PE}_{\mathrm{A}-\mathrm{SSI}}$ in the SSI-specific clusters combined and ambiguity-SSI-conjoined clusters combined; we also generated a 95\% prediction band for the effect of $\log \gamma_{1}$ on $\mathrm{PE}_{\mathrm{A}-\mathrm{SSI}}$ in an ambiguity-specific cluster (Figure 3 ). This reveals a significant heterogeneity in activation patterns across individual preferences toward ambiguity. In the four SSI-specific clusters, $\mathrm{PE}_{\mathrm{A}-\mathrm{SSI}}$ became significantly negative only for ambiguity-averse individuals and insignificant for ambiguity-tolerant individuals. In the ambiguity-specific cluster, the positive $\mathrm{PE}_{\mathrm{A}-\mathrm{SSI}}$ was significant only for ambiguity-tolerant individuals and insignificant for ambiguity-averse individuals. In the ambiguity-SSI-conjoined regions, $\mathrm{PE}_{\mathrm{A}-\mathrm{SSI}}$ was positive for ambiguity-tolerant individuals and negative for individuals high in ambiguity aversion. Despite a high positive correlation between $\gamma_{1}$ and $\gamma_{3}, \mathrm{PE}_{\mathrm{A}-\mathrm{SSI}}$ in all of these regions did not correlate with $\gamma_{3}$, suggesting that the modulating effect of the uncertainties generated through the lack of information is not influenced by individual preferences toward SSI.

Figure 3 depicts another way to visualize the relationships between preferences toward ambiguity and brainactivation patterns. Ambiguity-tolerant and ambiguity- 


\section{Ambiguity-Specific Regions (Logical Combination: $A \& \sim R \& \sim S S I$ \& C)}
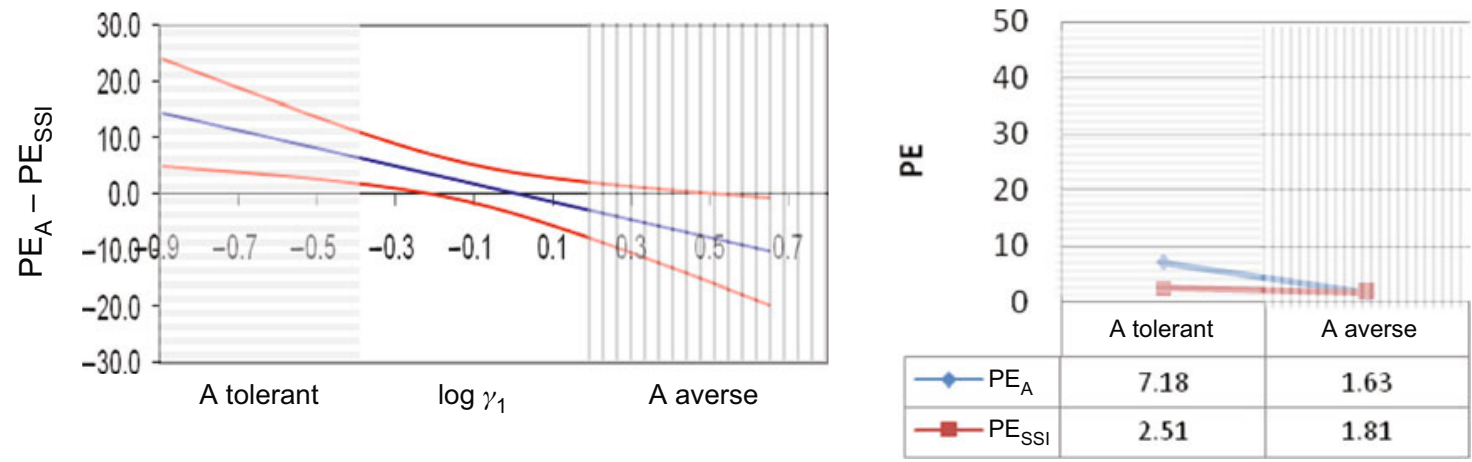

SSI-Specific Regions (Logical Combination: SSI \& R \& A \& C)
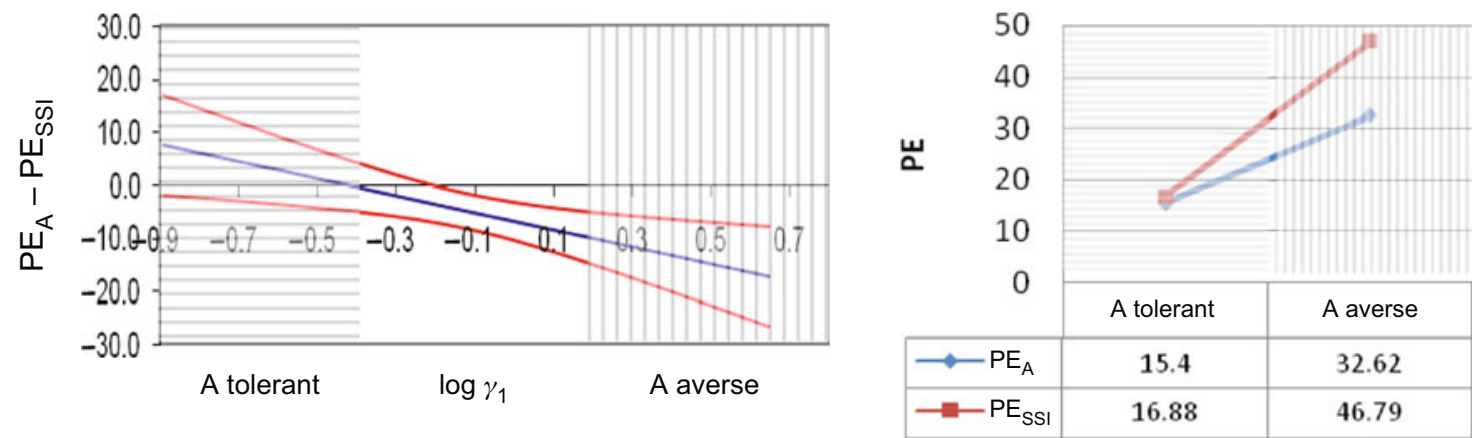

Ambiguity-SSI-Conjoined Regions (Logical Combination: [A \& SSI] \& R \& C)
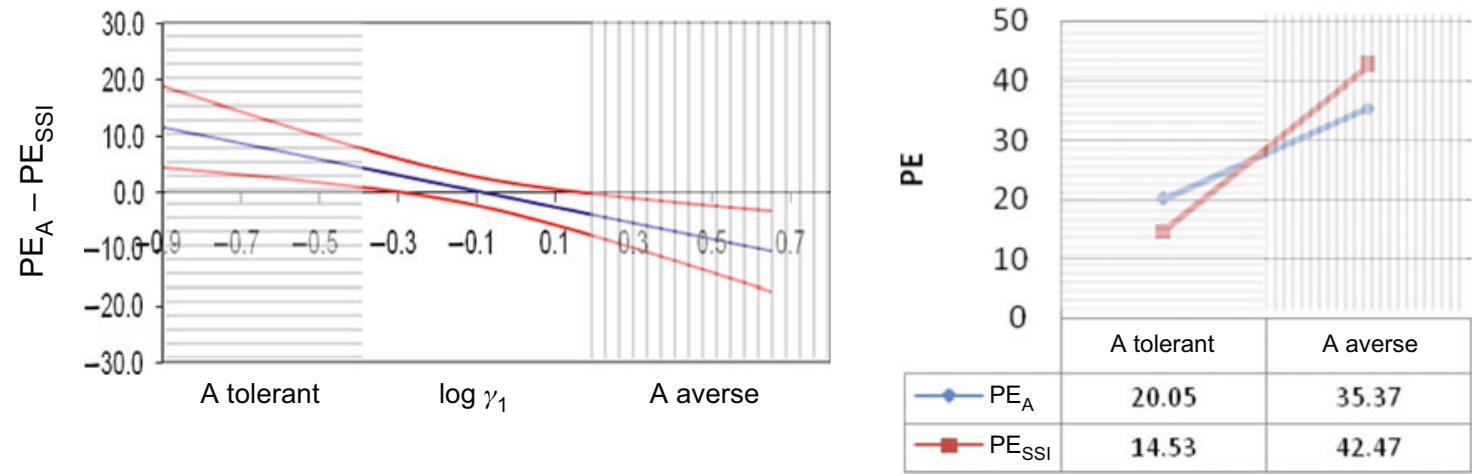

Figure 3. Predicting brain activation in ROIs from individual preferences toward ambiguity. The left panels depict the predicted main effect of $\log \gamma_{1}$ on $\mathrm{PE}_{\mathrm{A}-\mathrm{SSI}}$ and a $95 \%$ prediction band in ambiguity-specific, sample-space-ignorance (SSI) specific, and ambiguity-SSI-conjoined clusters. The right panels depict the interaction between the mean $\mathrm{PE}_{\mathrm{A}}$, the mean $\mathrm{PE}_{\mathrm{SSI}}$, and preferences toward ambiguity (ambiguity tolerance, $\log \gamma_{1}<-0.42$, vs. ambiguity aversion, $\log \gamma_{1}>$ 0.17). Horizontal stripes represent a region of ambiguity tolerance, and vertical stripes represent a region of ambiguity aversion.

averse individuals were defined as those $1 S D$ below and above the means for $\gamma_{1}$ (Cohen \& Cohen, 1983). The means of $\mathrm{PE}_{\mathrm{A}}$ and $\mathrm{PE}_{\mathrm{SSI}}$ for ambiguity-averse $\left(\log \gamma_{1}>\right.$ $0.17)$ and ambiguity-tolerant $\left(\log \gamma_{1}<-0.42\right)$ individuals in all ROIs were plotted when we detected a significant correlation between economic preferences and $\mathrm{PE}_{\mathrm{A}-\mathrm{SSI}}$ Recall that the interaction effect of preferences toward ambiguity on $\mathrm{PE}_{\mathrm{A}-\mathrm{SSI}}$ was significant.
Figure 3 illustrates that ambiguity-specific activation in the LaINS was driven primarily by the ambiguity-tolerant individuals. In addition, SSI-specific activation in the four SSI-specific clusters was driven by the ambiguity-averse individuals. Moreover, activation in the six ambiguitySSI-conjoined regions for ambiguity-tolerant individuals was stronger under the ambiguity than under the SSI condition, whereas for ambiguity-tolerant individuals, the 
activation was stronger under the SSI condition. However, ambiguity-averse individuals activated these regions more strongly than ambiguity-tolerant individuals. Overall, according to the $95 \%$ confidence intervals, activation patterns of ambiguity-tolerant individuals significantly differed from the activation patterns of ambiguity-averse individuals. Most importantly, the activation patterns of ambiguity-averse individuals were consistent with the reductive approach of modern decision-making theories, because SSI induced either more activation than ambiguity or the same level of activation as ambiguity, implying that activation for ambiguity was subsumed under activation for SSI. However, the activation patterns of the ambiguity-tolerant individuals violated the reductive viewpoint, because ambiguity induced more activation than SSI in some brain regions.

\section{DISCUSSION}

The reductive framework for decision making under uncertainty predicts more activation for SSI than for ambiguity during the valuation stage. This outcome emerged only for the ambiguity-averse individuals in regions shared for processing MI (primarily, the lateral prefrontal cortex). In these same regions and in the ambiguity-specific region (LaINS), the ambiguity-tolerant individuals showed more activation for ambiguity than for SSI, which is inconsistent with the reductive viewpoint. Consequently, the reductive viewpoint was supported for the ambiguity-averse individuals but disconfirmed for the ambiguity-tolerant individuals. This finding calls for new decision theories that can explain and predict decision making by ambiguity-tolerant individuals.

\section{MI-Conjoined Neurobiological Network}

The six ambiguity-SSI-conjoined regions that were modulated by ambiguity tolerance were primarily in the bilateral middle frontal gyrus, which is associated with numerous cognitive functions. With regard to decision making, one suggestion is that they are associated with the selection of a task context - namely, what information is relevant for a decision and in what context that information should be evaluated (Brass \& von Cramon, 2004). These regions have also been associated with deliberative rather than intuitive decision making (Kuo, Sjöström, Chen, Wang, \& Huang, 2009) and with working memory (Wager \& Smith, 2003). Therefore, the recruitment of these regions in the present study may reflect online storage of information that varies according to degree of uncertainty in different environments and information processing demands that vary with individual decision-making style (which might correlate with individual tolerance toward ambiguity). For instance, ambiguity-averse individuals may attempt to arrive at a decision via deliberative processing, therefore activating these regions more under SSI, when uncertainty is greatest (consistent with the reductive viewpoint). In contrast, ambiguity-tolerant individuals may employ more intuitive and less deliberative processing, activating these regions only for storage of information available about the environment (more under ambiguity than under SSI).

\section{Ambiguity-Specific Network}

Ambiguity uniquely activated the LaINS, and neuroticism was negatively correlated with insula activation. The anterior insula was activated for decision making under risk and ambiguity (Rushworth \& Behrens, 2008) and under higher levels of uncertainty (Rolls, 2008) or with subjective uncertainty (Feinstein, Stein, \& Paulus, 2006). Therefore, the left insula activation is not surprising, given that ambiguity is associated with imprecise probabilities. The negative correlation with neuroticism is unexpected in light of other findings that the right insula is associated with higher levels of neuroticism (Paulus, Rogalsky, Simmons, Feinstein, \& Stein, 2003). However, in most studies on the role of the insula in decision making, effects in the right or bilateral insula have been reported, with the association with neuroticism only in the right hemisphere. The fact that only the left insula was activated in the present study may point to a somewhat different role from that described in other studies.

Craig (2005) proposed that the left insula is associated with parasympathetic autonomic responses (rather than with sympathetic responses, as is the right insula). The parasympathetic system is associated with behavior that does not require an immediate response. Recall that selective activation in the LaINS was driven by ambiguitytolerant individuals. Possibly, the LaINS response reflects a tendency to rest and repose prior to making a response and may be important for intuitive decision making, which may be preferred by ambiguity-tolerant individuals under ambiguity. Ambiguity-averse individuals, on the other hand, may rely more on deliberative processing, which may not require pausing before starting calculations.

Interestingly, the selective activation in LaINS under ambiguity was not reported in Hsu et al. (2005) as distinguishing between ambiguity and risk. We attribute the discrepancy between our results and those reported by Hsu et al. to several important distinctions between designs used in our studies. First, we ensured that the type of gamble was not confounded with the probability splits, whereas in Hsu et al. all ambiguous gambles had the same split (the subjective probabilities associated with winning and losing were always $50 \%$ ), whereas the splits for risky gambles were varied. Thus, in a sense, Hsu et al. contrasted a series of risky gambles with the same ambiguous gamble. If the participants recognized that they were given the same ambiguous gamble time after time, they would not necessarily rely each time on intuition to evaluate it. Second, in our study, we used only card gambles, whereas Hsu et al. pooled together several conditions (card gambles, knowledge questions, and an informed-opponent condition). Therefore, our design models the risk condition more carefully: The participants were given the exact probabilities of winning associated with each card. Whereas in both the knowledge and the informed-opponent treatments of Hsu et al., even though the participants had a better knowledge about the environments classified as risky than they had about the environments classified as ambiguous, under risk they still had to rely on their subjective estimation of the underlying probabilities (what, in our terminology, defines ambiguity). Therefore, although the contrasts used in Hsu et al. 
could inform on how environments with less information differ from environments with more information, they do not necessarily describe the difference between the ambiguity and risk conditions.

\section{SSI-Specific Network}

SSI uniquely activated bilateral IPL, the ACC, and the left OFC, but recall that preferential activation in these regions was driven by ambiguity-averse individuals. In contrast to the LaINS activation for ambiguity, the SSI-specific network is more associated with higher level cognitive control and evaluative processes (possibly associated with more deliberative processing). For example, bilateral IPL may support cognitive processes related to sample space partitioning (such as recalling already seen cards and creating a mental map of the sample space; Piazza \& Dehaene, 2004), evaluation of probabilities associated with each possible outcome (Piazza \& Dehaene, 2004), expectation of losses (Dickhaut et al., 2003), or integration of numerical information (Vickery \& Jiang, 2009). Left lateral OFC activation may occur when processing negative reinforcers (Kringelbach \& Rolls, 2004) or negative reward information (Liu, Wang, Corbly, Zhang, \& Joseph, 2006), when insufficient information is available to make a decision about action (Elliott, Dolan, \& Frith, 2000), and is associated with anticipation anxiety (Chua, Krams, Toni, Passingham, \& Dolan, 1999), all of which might be expected under SSI. The ACC likely plays an evaluative role during SSI. It is implicated in error monitoring, response selection, and conflict resolution (Rushworth \& Behrens, 2008; Walton \& Mars, 2007), as well as flexibly engaging compensatory strategies during decision making (Botvinick, Braver, Barch, Carter, \& Cohen, 2001), which would be particularly important in unstable environments like SSI. Of all the uncertain environments explored here, SSI is the most unstable and would make the greatest demands on flexible behavior during decision making.

Overall, our results suggest that simple availability/ unavailability of information is not likely to fully describe the effect of uncertainty on valuation. Different types of uncertainty generated by MI recruit distinct neural substrates and thereby affect valuation differently, and individual preferences toward ambiguity interact with modulating effects of different types of MI environment.

The present analyses and interpretations are based on the assumptions that both psychological processes and brain functions can be reduced to discrete components and that specific brain areas/networks are mapped onto psychological processes in a one-on-one fashion. These assumptions apply to many psychological theories, not only decision making under uncertainty; however, they may not necessarily be true. Still, if we are interested in comparing the goodness of fit of different alternatives, given these assumptions - the approaches of reductive decision-making theories and the logical combination of brain-activation patterns - we can conclude that the fullhierarchy hypothesis does not fit the data for ambiguitytolerant individuals. A better understanding of the neural basis of decision making under uncertainty will require consideration of the variation in individual levels of tolerance for different types of uncertainty.

\section{AUTHOR NOTE}

This research was supported by Grants P50 DA005312, R01 HD052724, and P20 RR015592 from the Research Foundation of the University of Kentucky and from the National Institutes of Health. We thank Christine Corbly for her assistance. Correspondence concerning this article should be addressed to H. Pushkarskaya, Department of Agricultural Economics, University of Kentucky, 317 C.E. Barnhart Bldg., Lexington, KY 40546-0276 (e-mail: helen.pushkarskaya@uky.edu).

\section{REFERENCES}

BARON, J., \& Frisch, D. (1994). Ambiguous probabilities and the paradoxes of expected utility. In P. Ayton \& G. Wright (Eds.), Subjective probability (pp. 273-294). Chichester, U.K.: Wiley

Botvinick, M. M., Braver, T. S., Barch, D. M., Carter, C. S., \& Cohen, J. D. (2001). Conflict monitoring and cognitive control. Psychological Review, 108, 624-652. doi:10.1037/0033-295X.108.3.624

Brass, M., \& von Cramon, D. Y. (2004). Selection for cognitive control: A functional magnetic resonance imaging study on the selection of task-relevant information. Journal of Neuroscience, 24, 8847-8852. doi:10.1523/JNEUROSCI.2513-04.2004

Camerer, C., \& Weber, M. (1992). Recent developments in modeling preferences: Uncertainty and ambiguity. Journal of Risk \& Uncertainty, 5, 325-370. doi:10.1007/BF00122575

Chua, P., Krams, M., Toni, I., Passingham, R., \& Dolan, R. (1999). A functional anatomy of anticipatory anxiety. NeuroImage, 9, 563-571. doi:10.1006/nimg.1999.0407

Cohen, J., \& Cohen, P. (1983). Applied multiple regression/correlation analysis for the behavioral sciences ( $2 \mathrm{nd}$ ed.). Hillsdale, NJ: Erlbaum.

CrAIG, A. D. (2005). Forebrain emotional asymmetry: A neuroanatomical basis? Trends in Cognitive Sciences, 9, 566-571. doi:10.1016/ j.tics.2005.10.005

Dickhaut, J., McCabe, K., Nagode, J. C., Rustichini, A., Smith, K., \& PARdo, J. V. (2003). The impact of the certainty context on the process of choice. Proceedings of the National Academy of Sciences, 100, 3536-3541. doi:10.1073/pnas.0530279100

Elliott, R., Dolan, R. J., \& Frith, C. D. (2000). Dissociable functions in the medial and lateral orbitofrontal cortex: Evidence from human neuroimaging studies. Cerebral Cortex, 10, 308-317.

EllsberG, D. (1961). Risk, ambiguity, and the Savage axioms. Quarterly Journal of Economics, 75, 643-669.

Feinstein, J. S., Stein, M. B., \& Paulus, M. P. (2006). Anterior insula reactivity during certain decisions is associated with neuroticism. Social, Cognitive, \& Affective Neuroscience, 1, 136-142. doi:10.1093/ scan/ns1016

Fox, C. R., \& Rottenstreich, Y. (2003). Partition priming in judgment under uncertainty. Psychological Science, 14, 195-200. doi:10.1111/ 1467-9280.02431

Fox, C. R., \& TVErsky, A. (1995). Ambiguity aversion and comparative ignorance. Quarterly Journal of Economics, 110, 585-603. doi: $10.2307 / 2946693$

Hertzog, C., \& Rovine, M. (1985). Repeated-measures analysis of variance in developmental research: Selected issues. Child Development, 56, 787-809.

Hsu, M., Bhatt, M., Adolphs, R., Tranel, D., \& Camerer, C. F. (2005). Neural systems responding to degrees of uncertainty in human decision-making. Science, 310, 1680-1683. doi:10.1126/science .1115327

Huettel, S. A., Stowe, C., Gordon, E., Warner, B., \& Platt, M. (2006). Neural signatures of economic preferences for risk and ambiguity. Neuron, 49, 765-775. doi:10.1016/j.neuron.2006.01.024

Jenkinson, M., Bannister, P., Brady, M., \& Smith, S. (2002). Improved optimization for the robust and accurate linear registration and motion correction of brain images. NeuroImage, 17, 825-841. doi:10.1016/S1053-8119(02)91132-8

Joseph, J. E., Partin, D. J., \& Jones, K. M. (2002). Hypothesis testing for selective, differential, and conjoined brain activation. Jour- 
nal of Neuroscience Methods, 118, 129-140. doi:10.1016/S0165 $-0270(02) 00122-X$

Kringelbach, M. L., \& Rolls, E. T. (2004). The functional neuroanatomy of the human orbitofrontal cortex: Evidence from neuroimaging and neuropsychology. Progress in Neurobiology, 72, 341-372. doi:10.1016/j.pneurobio.2004.03.006

Kuo, W.-J., Suöström, T., Chen, Y.-P., WAng, Y.-H., \& HuAng, C.-Y. (2009). Intuition and deliberation: Two systems for strategizing in the brain. Science, 324, 519-522. doi:10.1126/science.1165598

Liu, X., Wang, H., Corbly, C. R., Zhang, J., \& Joseph, J. E. (2006). The involvement of the inferior parietal cortex in the numerical Stroop effect and the distance effect in a two-digit number comparison task. Journal of Cognitive Neuroscience, 18, 1518-1530. doi:10.1162/ jocn.2006.18.9.1518

Mintun, M. A., Fox, P. T., \& Raichle, M. E. (1989). A highly accurate method of localizing regions of neuronal activation in the human brain with positron emission tomography. Journal of Cerebral Blood Flow \& Metabolism, 9, 96-103.

Modica, S., \& Rustichini, A. (1994). Awareness and partitional information structures. Theory \& Decision, 37, 107-124. doi:10.1007/ BF01079207

Modica, S., \& Rustichini, A. (1999). Unawareness and partitional information structures. Games \& Economic Behavior, 27, 265-298.

Nichols, T., Brett, M., Andersson, J., Wager, T., \& Poline, J. B. (2005). Valid conjunction inference with the minimum statistic. NeuroImage, 25, 653-660. doi:10.1016/j.neuroimage.2004.12.005

Paulus, M. P., Rogalsky, C., Simmons, A., Feinstein, J. S., \& Stein, M. B. (2003). Increased activation in the right insula during risktaking decision making is related to harm avoidance and neuroticism. NeuroImage, 19, 1439-1448. doi:10.1016/S1053-8119(03)00251-9

Piazza, M., \& Dehaene, S. (2004). From number neurons to mental arithmetic: The cognitive neuroscience of number sense. In M. Gazzaniga (Ed.), The cognitive neurosciences (3rd ed.). Cambridge, MA: MIT Press.

Platt, M. L., \& Huettel, S. A. (2008). Risky business: The neuroeconomics of decision making under uncertainty. Nature Neuroscience, 11, 398-403. doi: $10.1038 / \mathrm{nn} 2062$

QuigGin, J. (1993). Generalized expected utility theory: The rank dependent model. Boston: Kluwer.

Rangel, A., Camerer, C., \& Montague, P. R. (2008). A framework for studying the neurobiology of value-based decision making. Nature Reviews Neuroscience, 9, 545-556. doi:10.1038/nrn2357

Rolls, E. T. (2008). Memory, attention, and decision-making: A unify- ing computational neuroscience approach. Oxford: Oxford University Press.

Rushworth, M. F. S., \& Behrens, T. E. J. (2008). Choice, uncertainty and value in prefrontal and cingulate cortex. Nature Neuroscience, 11, 389-397. doi:10.1038/nn2066

Sмiтн, S. M. (2002). Fast robust automated brain extraction. Human Brain Mapping, 17, 143-155.

Smithson, M. (1999). Conflict aversion: Preference for ambiguity vs. conflict in sources and evidence. Organizational Behavior \& Human Decision Processes, 79, 179-198. doi:10.1006/obhd.1999.2844

Smithson, M., Bartos, T., \& TaKemura, K. (2000). Human judgment under sample space ignorance. Risk, Decision \& Policy, 5, 135-150.

Tversky, A., \& Kahneman, D. (1992). Advances in prospect theory: Cumulative representation of uncertainty. Journal of Risk \& Uncertainty, 5, 297-323. doi:10.1007/BF00122574

VICKERY, T. J., \& JiANG, Y. V. (2009). Inferior parietal lobule supports decision making under uncertainty in humans. Cerebral Cortex, 19, 916-925. doi:10.1093/cercor/bhn140

von Neumann, J., \& Morgenstern, O. (1944). Theory of games and economic behavior. Princeton, NJ: Princeton University Press.

WAGER, T. D., \& SMITH, E. E. (2003). Neuroimaging studies of working memory: A meta-analysis. Cognitive, Affective, \& Behavioral Neuroscience, 3, 255-274. doi:10.3758/CABN.3.4.255

Walton, M. E., \& Mars, R. B. (2007). Probing human and monkey anterior cingulate cortex in variable environments. Cognitive, Affective, \& Behavioral Neuroscience, 7, 413-422. doi:10.3758/CABN.7.4.413

Xiong, J., GaO, J.-H., Lancaster, J. L., \& Fox, P. T. (1995). Clustered pixels analysis for functional MRI activation studies of the human brain. Human Brain Mapping, 3, 287-301.

\section{NOTE}

1. $\gamma_{1}$ and $\gamma_{3}$ were distributed lognormally in our sample.

\section{SUPPLEMENTAL MATERIALS}

Additional notes on the procedure, additional significant brain activation patterns, and instructions and a quiz given to our participants may be downloaded from http://cabn.psychonomic-journals.org/content/ supplemental.

(Manuscript received September 29, 2009; revision accepted for publication March 24, 2010.) 